\title{
Salay-siday: Mga karanasan ng pagbangon pagkatapos ng sakuna
}

Galit, Gilbert $\bowtie$

Leyte Normal University, Philippines (gilfaculty27@gmail.com)

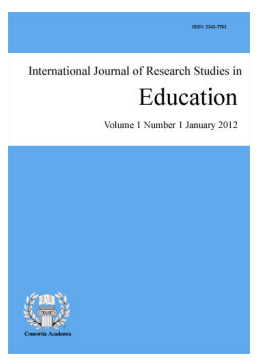

Accepted: 30 May 2021

ISSN: 2243-7703 Online ISSN: 2243-7711

OPEN ACCESS

\section{Abstract}

Literature is a reflection of life, adventure, emotions, and experiences. One of these is poetry that has always been used in conveying the realities of life. This study examines the different emotions and experiences reflected in literary works written after the devastation of super typhoon Yolanda (International name, Haiyan). This is a qualitative research with a content analysis design aimed to elicit the different emotions and experiences of the victims that can be seen in the poems. A thematic analysis was used to organize and interpret the data. The poems were taken from the book, Lunop: Haiyan Voices and Images published by the Samar-Leyte Heritage Society, Inc. Ten (10) poems were selected and analyzed in response to the research objectives. Based on the analysis done, the phrases in the poems used several figurative speeches and portray various persona such as a sympathetic person who cares about the welfare of the victims, a narrators and some are observers. The poems also contain different emotions representing the personas. Ultimately, the researcher identifies the experiences of the victims reflected in the literary pieces. It was concluded that poems contain intense emotions and experiences of suffering and recovery. With these, the researcher highly recommends to conduct further studies focusing on the association of the author's life with his works in order to better see the authenticity of the experiences and feelings of the poems written after the disaster.

Keywords: Yolanda; siday; emotions; experiences; recovery 


\section{Salay-siday: Mga karanasan ng pagbangon pagkatapos ng sakuna}

\section{Introduksiyon}

Ang panitikan ay salamin ng katotohanan at daluyan ng damdamin at karanasan. Ihinahayag nito ang larawan ng isang lipunan na siyang pangunahing saligan sa pag-unawa sa kultura, gawi at pamumuhay ng isang pangkat. Bumubuo ito ng mga kaisipan na nagpapahayag ng isang kaparaanan na likha ng guniguni at sagimsim na salig sa buhay na aktuwal na nagaganap o maaaring maganap.

Ang tula ay buhay at may sariling mundo. Mundong nagsisilbing anino ng realidad na nag-aanyo sa iba't ibang porma at katauhan upang ilahad ang isang kaisipang natatangi sa di-tahasang paraan. Sa kasalukuyan, isa sa mga pinakapopular na pamamaraan ng masining na pagpapahayag ang pagsulat ng tula na binihisan sa makabagong panahon ng bagong anyo. Naglalaman ito ng matalinhagang pagpapahayag na naglalarawan ng karanasan at damdamin ng isang manunulat at/o manunula. Noon pa man ay gamitin na ang tula at iba pang genre sa pagsasabuhay sa mga pangyayaring pangkasaysayan at pangkasalukuyan. Nariyan ang mga tula nina Rizal, Bonifacio, Baltazar at marami pang iba na naglalahad ng mga katotohan bilang repleksiyon ng pakikibaka at pagsulong ng isang tao o maging ng lipunan. Libo-libong koleksiyon ng mga tula ang nabubuo mula sa iba't ibang penomenon. Hindi lamang nito naisasadokumento ang mga pangyayari, higit sa lahat ay naipahahayag nito ang kaisipan sa madamdaming paraan na nagpapalitaw ng buhay at kaluluwa.

Nobyembre 8, 2013 ay binulabog ang buong mundo lalo na ang Eastern Visayas nang nanalanta ang pinakamalakas na bagyo na naitala sa kasaysayan, ang bayong Yolanda o Haiyan (international name). May dala itong wind speed na humigit-kumulang sa 300 kilometer per hour $(\mathrm{km} / \mathrm{ph})$ na nagdala ng dambuhalang daluyong na sumira sa libo-libong kabuhayan, imprastraktura at higit sa lahat - buhay. Sa ulat ni Ranada (2013), tinatayang nasa 9.46 Bilyong piso ang pinsalang naidulot nito at humigit sa 10,000 ang mga namatay habang may ilan pang naitalang nawawala. Ang malagim na karanasang ito ng mga taga-Eastern Visayas ay isang pangyayaring sumukat sa katatagan at pagkakaisa hindi lamang ng mga Pilipino kundi ng buong mundo.

Ang kulturang Pilipino ay nagpapamalas ng matatag na paniniwala sa Diyos. Isa ito sa mga lakas na pinanghawakan ng mga biktima sa pakikipagsapalaran at paghakbang tungo sa pagbangon mula sa pagkalugmok. Marami ang nawalan ng tirahan at kabuhayan, maraming buhay ang nawala, pangarap at pag-asa. Iba-ibang pananaw ang lumitaw at iba-ibang hamon ang pinagdaanan. Sa unti-unting pagbangon ng mga biktima, unti-unti ring nabuo ang kasaysayang tumatak sa puso’t isipan ng lahat. Mula sa unang araw ng kalamidad hanggang sa kasalukuyan, ang gayong panahon ay nagbigay ng lakas sa mga biktima upang ibahagi ang kani-kaniyang kuwento. Nagbunga ito ng iba't ibang panitikan mula sa mga testimonya ng tao na nagbigay inspirasyon sa pagbuo ng mga tula na nagpapahayag ng emosyon na tumatakbo sa kanilang mga puso, isip at kaluluwa (Alegre, 2015).

Ipinaliwanag ni Santiago (1989) na ang tunay na kahulugan ng panitikan ay yaong pagpapahayag ng damdamin, panaginip at karanasan ng sankatauhang nasusulat sa maganda, makahulugan at masining na pahayag. Dagdag pa ni Villafuerte (2000), ang panunuring pampanitikan ay hindi lamang nagsusuri o nagbibigay kahulugan sa mga nagaganap sa daigdig kundi ito ay isang paraan ng pagsusuri sa kabuuan ng tao - kaniyang anyo, ugali, kilos, paraan ng pagsasalita, at maging ng kaniyang pakikipag-ugnayan sa kaniyang kapuwa at sa lipunang kaniyang kinabibilangan.

Inilarawan ni Leyson na binanggit sa pag-aaral ni Rio (2013), ang tula ay paraan ng pagpapahayag ng kaisipan at karanasan sa pamamagitan ng madamdamin at masining na pananalita. Kaya, napakalaki ng kaugnayan ng buhay ng may-akda sa kaniyang obra. Kung susuriing mabuti, itinatala ng makata ang kaniyang buhay sa kaniyang panulat na kung minsan ay hindi niya napapansin o kaya ay itinatago lamang niya sa kaniyang mga panulat.

68 Consortia Academia Publishing (A partner of Network of Professional Researchers and Educators) 
Malinaw na ipinaliwanag ni San Juan (1995) na kinakailangan ng kritiko o mambabasa na magsuri upang makatulong sa lalong ikaliliwanag ng panitikan at gayon ng manunulat. Mabilis at mas madaling matukoy at makilala ang kaisipan, damdamin at larawan ng isang akda dahil sa mga disenyo at teorya na magagamit sa pagsusuri. Sa tulong nito, mas nabibigyan ng tumpak na pagpapakahulugan ang isang akda sa pamamagitan ng paghihimay-himay sa mga elemento na ginamit ng may-akda.

Upang mas bigyang-linaw ang pag-unawa sa pagsusuri ay inilahad din sa pag-aaral na ito ang mga pag-aaral na nagpapaliwanag at naglalahad ng mga tuklas na impormasyon hinggil sa pagsusuri ng tula upang pagtibayin ang kasalukuyang pag-aaral.

Napatunayan sa pag-aaral ni Rio (2013) na ang mga tula ay naglalahad ng mga katotohanan at damdamin. Sa pagsusuri na kaniyang ginawa sa mga piling tula ni Amado V. Hernandez nakita niya na ang mga akdang ito ay nagsisiwalat ng iba't ibang temang may pagpapahalaga gaya ng pagkamakabayan, pagkamakatotohanan at pagkamakatarungan tungo sa kamalayang pangmamamayan. Dagdag pa ni Cericos (2011) na binanggit sa parehong pag-aaral, sinasabi na mabuting batayang bahagi ng isang akda ay hinugot mula sa sariling karanasan ng manunulat at hindi nilikom lamang sa pahina ng mga aklat. Ito ay kaalamang pinipili ng karanasan, karanasang nagbigay kaalaman sa pagpapaigting ng damdamin at nagpapakislot ng isip at nakapagpapaunawa sa katotohanan.

Gayon din sa mga tuklas ni Arsolon (2012) sa kaniyang pag-aaral na "El Filibusterismo ni Rizal: Mimesis na Sosyo-Politikal," nagsasaad na isinusuot ng isang lipunan ang pangit na larawang panlabas nito ngunit maganda naman sana ang nasa loob nito ngunit nilaspatangan lamang ng mga humahawak. Patunay lamang na ang tula ay isang kasangkapang literari-kultural na nagpapahiwatig ng maraming kaisipan, karanasan at damdamin.

Sa pag-aaral ni Macan (2000), natuklasan niyang ang mga piling romantikong tula ni Jose Corazon de Jesus ay nagpadarama ng mga konseptong makatao sa pamamagitan ng mga simbolismo at paralelismo. Simbolismo dahil gumamit siya ng mga bagay na akmang-akma sa mga kaugalian at katangian ng isang tao. May kaugnayan ito sa kasalukuyang pag-aaral sapagkat binibigyang pansin din dito ang paggamit ng mga simbolismo sa pagpapahayag ng mga karanasan at damdamin ng may-akda, sa sarili at sa lipunan.

Totoong ang bawat akda ay may kaniya-kaniyang pananaw. Iba-ibang karakter at paglalarawan ng damdamin at karanasan. Ito ay isang uri ng pagsasalaysay na pinalilitaw ang kagandahan ng paggamit ng wika upang maikubli ang mga damdaming marahas, masalimuot at gayundin minsan ay masaya. Upang palitawin ang mga kaisipan, karanasan at damdamin ng isang tula, ang kasalukuyang pag-aaral ay gumamit ng teoryang mimetiko upang ipahayag ang pagsasabuhay ng katotohanan sa pamamagitan ng pagsusuri. Dito masasalamin ang panggagagad ng isang panitikan sa realidad at tunay na kalakalan sa lipunan na naglalaman ng iba't ibang larawan ng karanasan at damdamin. Binigyang-linaw ni Catipay (2013) mula sa aklat ni Aristotle na "On Interpretation" na ang teoryang mimetiko ay replika ng totoong buhay. Ipinaliliwanag dito na ang lahat ng bagay sa mundo ay sumisimbolo sa totoong karanasan ng isang tao sa lipunang kaniyang ginagalawan.

Ang mga gayong paglikha ay bagong tala ng kasaysayan at kontribusyon sa panitikan. Bagaman ilang taon na ang lumipas ay mababakas pa rin ang hagupit at pait ng karanasang kanilang pinagdaanan. Nanatili ang takot at pangamba maging sa kasalukuyan lalo pa't nag-iwan ito ng malalim na sugat sa puso ng mga biktima. Gayunpaman, ang pagkakabuo ng mga akdang ito ay isang magandang paalaala na minsa'y nasubok ang katatagan ng bawat isa. Dahil sa pagsibol ng panitikan hinggil sa iba't ibang karanasan ay nagawa nitong maipakita sa susunod na henerasyon ang kahalagahan ng pag-asa at pagkakaisa. Iba't ibang kuwento, karanasan at damdamin ang nilalayong tukuyin sa pag-aaral na ito sa pamamagitan ng pagsusuri sa mga tulang naisulat pagkatapos ng Yolanda. Layon nitong mailarawan ang iba’t ibang damdamin at pagpapahalaga sa gitna ng matinding pagsubok gaya ng Yolanda. 


\subsection{Kaligirang teoretikal}

Isang mahalagang lapit teoretikal sa pag-unawa sa ugnayan ng panitikan at kultura upang mailarawan ang panlipunang estruktura ay ang Structuralism (1950's-1960's) na hinalaw ni Claude Levi-Strauss sa teoryang structural linguistics na binuo ng isang lingguwistang Swiss na si Ferdinand de Saussure (Mambrol, 2016). Sa teoryang ito, binibigyang-pansin ang mga di-lantad na kayarian ng wika na may tiyak na epekto sa pag-uugali, lipunan at kultura ng isang pangkat na inilalarawan sa akda. Ayon pa kay Saussure na binanggit sa artikulo ni Mambrol (2016), ang anumang wika ay binuo sa paraang ang lahat ng elemento nito ay magkakaugnay nang di-arbitraryo, regular, at nakasalalay sa mga tuntunin. Ang isang kompetent na tagapagsalita/tagapagsulat nito ay gumagamit ng wika ayon sa mga umiiral na tuntunin subalit hindi namamalayan. Tungkulin ng pagsusuri na alamin ang mga pumapailalim na estruktura, kasama na ang mga transpormasyong nagaganap na konektado sa kayarian ng iba’t ibang ekspresiyon.

Sa pag-aaral na ito, nailalapat ang konsepto ng teorya sa pagtukoy ng kaligiran ng wikang Waray bilang kasangkapan ng may-akda sa pagsasalin at paglalarawan ng mga karanasan ng persona sa mga siday (tawag sa panulaang Waray). Ang istilo at paraan ng paghahabi ng bawat linya at pagpili ng mga salita sa pagsulat ay isang mahalagang salik upang lubos na mapalitaw ang mga nakakabit na karanasan, pakikipagsapalaran, damdamdamin at kaugalian. Hindi maikakailang ang wika at kultura ay magkabuhol na siyang saligan sa pagkilala sa kalikasan ng bawat isa. Ang teoryang structuralism ay paunang hakbang sa pagsusuri ng mga tula na naglalayong makapaglatag ng mga tumpak na kaisipan na nagpapatatag sa likas na katangian ng wika na makapaglarawan ng mga katotohanan tungkol sa mga gumagamit nito.

Dagdag pa, ang panitikan ay larawan ng ugnayan ng lipunan sa kaniyang kapaligiran na ipinaliliwanag sa teoryang Symbolic Interactionism ni Irving Goffman (1922-1982), isa sa mga pangunahing proponent ng teorya. Ayon sa kaniya, ang teoryang ito ay may maykrolebel na nakapokus sa ugnayan ng bawat isa sa loob ng isang lipunan. Ginagamitan ito ng pakikipagtalastasan kung saan ang bawat isa ay nagpapalitan ng ideya o pagpapakahulugan sa pamamagitan ng wika at simbolo - ito ay pinaniniwalaan na nagbibigay saysay sa pakikipagsalamuha ng tao sa lipunan (Redmond, 2015).

Sa pamamagitan ng symbolic interactionism mas nagkakaroon ng ugnayan ang panitikan at kultura dahil sa paraang ito naihahatid ang mga makabuluhang simbolikong kultural sa lipunan na agiging salamisim ng mga manunulat upang mailarawan ang gayong gawi ng lipunan. Naipararating ng panitikan ang mga nais nitong ihayag na katotohanan at dinamikong galaw ng lipunan gamit ang wika na may malawak na pagpapakahulugan at pagpapahayag. Dahil sa masining na pagsasalaysay ng manunulat ay nagkakaroon ng interaksiyon ang tao sa kaniyang lipunan sa pamamagitan ng mga likha nito. Dahil dito, nauunawaan ng mga mambabasa ang kultura ng isang manunulat at ng lipunang kaniyang kinabibilangan o kaya'y ang paraan ng pamumuhay ng isang lugar na inilalarawan.

Ayon sa mga teorista na sina Herman at Reynolds (1994), ang pananaw na ito ay kakikitaan ng makabuluhang ugnayan ng tao sa kaniyang lipunan at sa paghubog ng mga kulturang kanilang kinasasangkutan. Dahil dito, nagiging pang-araw-araw na gawi ng tao ang mga bagay-bagay na nakikisalamuha at kalimitan ay nagiging bahagi ng kultura ng isang lipunan. Sa karagdagan, ang panitikan sa teoryang ito ay isang sangkap sa isang makabuluhang talastasan sa loob ng lipunan dahil sa simbolong ginagamit, mga paglalarawan sa isang pangyayari o bagay at pagpapalitan ng kuro-kuro sa isang ideya (Egoy et al., 2019).

Sa pag-aaral na ito ay naipakikita ang iba't ibang karanasan ng mga tulang naisulat pagkatapos ng Yolanda. Sinuri ang mga napiling tula sa pamamagitan ng mga teoryang pampanitikang mimetiko at pormalistiko. Ang teoryang Mimetiko ay ideya na ang sining ay gumagaya sa katotohanan. Ang tagasuri o mambabasa ang nagbibigay kahulugan sa pampanitikang gawa na kopya o gaya sa kalikasan. Ang pagsusuring mimetiko ay nauukol sa kaugnayan ng tula sa kalikasan o sa buong mundo (Gleeson, 2018). Ipinakikita sa teoryang ito ang masusing pagkabuo at kritikal na pormulasyon para unawain ang ugnayan ng akda at katotohanan. 
Layunin ng teoryang ito na pumuna upang matukoy kung gaano kahusay ang isang pampanitikang gawa kaugnay sa tunay na mundo. Isang teoryang maaaring magpalawak at maisama ang mga pamamaraang kaugnay sa isprituwal at pagpapakahulugan, at kaugnayan ng imahen sa tao sa lahat ng oras maging sa kultura. Gayundin, ginamit bilang saligang teoretikal sa pag-aaral na ito ang teoryang pormalistiko. Ito ay isang teorya o uring kritisismong pampanitikan na nagbibigay-diin sa porma ng isang teksto at hindi sa nilalaman. Binibigyan ng markadong atensiyon ang estraktura, istilo at anyo ng pagsulat. Ginagamit ang teoryang ito dahil kaugnay nito ang kuwentong may nakapaloob na ginagamit sa pagsusuri.

Ang layunin ng panitikan ay iparating sa mambabasa ang nais niyang ipaabot gamit ang kaniyang tuwirang panitikan. Samakatuwid, kung ano ang sinasabi ng may-akda sa kaniyang panitikan ang siyang nais ipaabot sa mambabasa - walang labis at walang kulang. Walang simbolismo at hindi humihingi ng higit na malalim na pagsusuri't pag-unawa. Napakahalaga ng teksto sa paggamit ng teoryang pormalistiko sapagkat kinakilangang masuri ang tema at paksa ng akda, ang sensibilidad ng mga tauhan at pag-uugnayan ng mga salita, estruktura ng wika, metapora, imahen at iba pang elemento ng isang akda. Sa pagsusuri ng isang kuwento, kinakailangang masuri ang mga elementong nakapaloob sa akda tulad ng pangunahing tauhan, tema, tunggalian at tema.

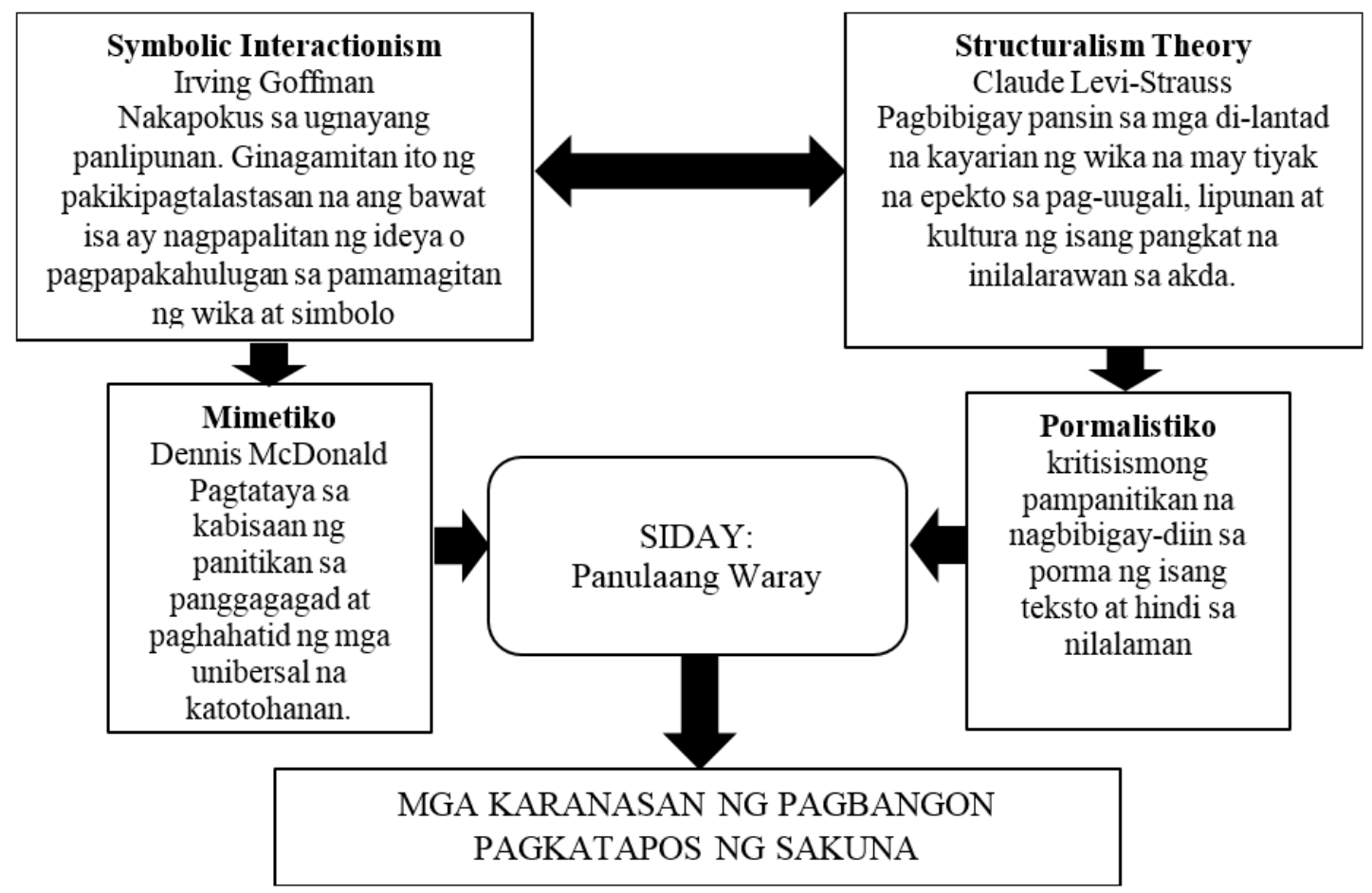

Figyur I. Balangkas Teoretikal ng Pag-aaral

Ang mga teoryang symbolic interactionism at structuralism ang mga saligan na ang wika at kultura ay may ugnayang kultural na nagiging batayan ng paglalarawan ng mga gawi, damdamin at karanasan ng isang tao at/o pangkat ng tao. Sa pamamagitan ng mga kritisismong pampananitikang mimetiko at pormalistiko ay sinuri ang mga akdang Waray na naging batayan upang palitawin ang mga karanasan ng pagbangon pagkatapos ng sakuna ng mga biktima ng bagong Yolanda o Haiyan.

\subsection{Paglalahad ng suliranin}

Layunin ng pananaliksik na ito na masuri ang iba't ibang damdamin at karanasan ng mga biktima ng Yolanda na makikita sa tula. Tinitiyak na masuri sa pananaliksik na ito ang mga sumusunod:

Estruktura ng mga tulang naisulat pagkatapos ng Yolanda batay sa; (a) tayutay (b) persona (c) simbolismo (d) tema;

$>\quad$ Damdaming nakapaloob sa mga siday na naisulat pagkatapos ng sakuna; at 
Karanasang nakapaloob sa mga siday na naisulat pagkatapos ng sakuna.

\section{Metodolohiya}

Ang pananaliksik na ito ay isang kuwalitatibong pag-aaral at gumamit ng content analysis upang bigyan ng pagpapakahulugan ang mga tulang naisulat pagkatapos ng sakuna. Sinuri at inilarawan ng mananaliksik ang mga natuklasan batay sa mga binanggit na mga layunin. Sampung (10) tula ang sinuri na pinili ayon sa kaangkupan at kahalagahan ng datos na naaayon sa layunin ng pananaliksik. Kinuha ang mga ito sa aklat na Lunop: Haiyan Voices ang Images na nilimbag ng Leyte-Samar Heritage Society, Inc. noong 2015. Ang lokal ng pag-aaral ay Eastern Visayas na may mga tulang naisulat pagkatapos ng sakuna. Sinuri ang estruktura ng mga ito ayon sa ginamit na mga elemento gaya ng tayutay, persona, simbolismo at tema. Nang matukoy na ang mga ito ay muling sinuri ang mga tula batay sa mga damdaming nakapaloob dito upang makita ang mga karanasang isinasalaysay sa mga tulang naisulat pagkatapos ng sakuna.

Ginamit ang tematikong analisis sa pag-aaral bilang paraan ng tritment sa mga datos na natukoy. Ang bawat magkakaugnay na paksa, kaisipan at konteksto ay pinagsama-sama upang palitawin ang mga natatanging tema. Ang pag-uuri sa mga temang ito ay nakabatay sa mga ideyang ipinahihiwatig. Upang matiyak na balido at kapani-paniwala ang mga nalikom na datos, sinuri ang mga ito ng mga maaasahang tagasuri sa pamamagitan ng pagberipika gamit ang tseklist.

\section{Mga resulta at diskusyon}

Matutunghayan sa bahaging ito ang presentasiyon at pagpapakahulugan sa mga nakalap na datos kaugnay sa mga inilahad na mga layunin ng pag-aaral.

\subsection{Estruktura ng mga tulang naisulat pagkatapos ng Yolanda}

Sa pagsusuri sa nilalaman ng mga tula batay sa estruktura nito, nalaman ng mananaliksik ang mga pagpapakahulugan sa paggamit ng elemento ng tula upang ilarawan ang iba't ibang damdamin at karanasan ng mga biktima pagkatapos ng sakuna.

Tayutay - Ang tayutay ay isang pamamaraan ng paggamit ng wika na inililihis sa literal na pagpapahayag ang mga pangyayari at/o damdamin. Taglay nito ang makulay na pananalita upang palutangin ang kagandahan at sining ng wika na nagbibigay ng kariktan sa pagbuo ng tula. Hinahamon nito ang kakayahan sa kritikal at malikhaing pag-iisip ng mga mambabasa. Sa mga tulang naisulat pagkatapos ng sakuna, ginamit ang mga sumusunod na tayutay:

\section{Talahanayan I}

Mga ginamit na tayutay sa mga Siday

\begin{tabular}{ll}
\hline \multicolumn{1}{c}{ Siday (Tula) } & \multicolumn{1}{c}{ Tayutay } \\
\hline Ayaw Paurhi, Buligi an Nagkukuri & Pagbibigay Katauhan, Onomatopiya \\
Gugma, Paglimot ug iba pa nga mga Pinalaypay & Pagbibigay Katauhan \\
Han Pagbisita ni Apoy Yolanda & Pagbibigay Katauhan \\
Ikmat sa Dagkon Balud & Pagbibigay Katauhan, Pagmamalabis \\
& Pagtutulad \\
Liro & Pagtutulad \\
Nuche Buena Ala Yolanda & Pagbibigay Katauhan \\
Pag-iliw & Paralelismo \\
Sa Muling Pagsibol & Pagbibigay Katauhan, Pagmamalabis \\
Where u? & Pagpapalit-saklaw \\
Yolanda & Pagtawag \\
\hline
\end{tabular}

a.1. Pagbibigay Katauhan (Personification). Ang pagbibigay katauhan ay isang pamamaraan ng matalinghagang pagpapahayag na naglalarawan sa isang bagay sa pamamagitan ng pagbibigay katangian na gaya

72 Consortia Academia Publishing (A partner of Network of Professional Researchers and Educators) 
ng isang tao. Marami sa mga tulang naisulat ay gumamit ng ganitong uri ng tayutay gaya na lamang ng sa tulang "Noche Buena Ala Yolanda" sa mga linyang, "Nga gindagit san dagat." Dito ay binigyang katangian ng may-akda ang dagat na tulad ng isang taong tumangay ng isang bagay. Sa tula inilalarawan ang kalunos-lunos na pangyayari ng tangayin ng malaking alon ang libo-libong buhay na nagdulot ng malagim na pagkamatay ng marami. Gayundin, makikita sa tula ni Voltaire Oyzon na "Gugma, Paglimot Ug Iba pa nga Pinalaypay" ang linyang, "Katima pantukas hin atop ni "Ruby" na nagpapahayag din ng puwersahang pag-aalis ng mga bubong ng kabahayan sa kasagsagan ng delubyo. Ito ay pagpapakita ng bagsik at lakas ng bagyo na dumaan sa mga biktima. Ginamit din ni Oyzon ang tayutay na, "Han nangungunat pa la nga adlaw” upang mailarawan naman ang bagong pag-asa na sumilay sa mga biktima pagkatapos ng isang malagim na sakuna. Ang salitang nangungunat (umuunat) ay isang gawi na kadalasang ginagawa ng isang taong bagong gising pa lamang. Sa linyang ito ipinakikita ni Oyzon ang bagong unat na araw na simbolo ng pag-asa para sa lahat ng mga biktima. Sa tulang "Han Pagbisita ni Apoy Yolanda" kapansin-pansin din ang linyang, "Ha pagbisita ni apoy Yolanda." Ang linyang ito ay pagsasalaysay ng mga pangyayari sa pagbisita ng isang tao sa isang lugar o tahanan subalit hindi naging kaaya-aya ang kinahinatnan nito. Sa linyang ito ay binigyang katauhan ng may-akda ang bagyong Yolanda na inilarawan sa mga saknong na dahilan ng pagkasira ng mga kabahayan at maging ng maraming buhay.

a.2. Pagmamalabis (Exaggeration). Ginamit sa tula ang ganitong uri ng tayutay upang ilarawan ang mga pangyayari sa tula ng may kalabisan. Sa tula ginamit ang mga salitang, "Nagkarigumok (nagkagulo) an mga tawo", "bitbit (bitbit), danas (hila-hila) adton mga anak nga nagdadadlagan", "May puro kagis (sugat) an panit kay tumara sa kabanwaan." Sa mga pahayag na ito, inilarawan ng may-akda ang sitwasyon na naganap sa kasagsagan ng sakuna. Ang unang linya ang pagpapakita ng nangyaring kaguluhan, pagpapa-panic, at pagkabalisa ng mga biktima. Sa ikalawang linya naman ipinakikita ang sugatang mga katawan ng mga biktima ng sakuna habang tangay-tangay o bitbit ang mga anak. Isa itong malinaw na larawan tungkol sa sobrang hirap na pinagdaanan ng mga biktima. Sa tulang "Muling Pagsibol" ginamit din ang linyang, "Ulan at Hangin ginunaw ang dapa." Ang salitang ginunaw ay pagmamalabis sa pagsira ng bagyo sa daan-daang ari-arian, kabuhayan, kabahayan at maging buhay ng maraming biktima.

Pagtutulad (Simile). Ito ay isang uri ng tayutay na ginagamit bilang paraan ng paghahambing sa isang bagay sa isa pang bagay na may magkatulad na katangian. Sa tulang "Liro" ni Pundavela, makikita ang ganitong uri ng tayutay sa mga linyang, "An alas kwatro han kulop baga in gab-i," "Mamingaw, nagdudulom sugad-sugad hin ginsuyop han lunop an bulan ngan kabitun-an." Sa mga linyang ito ay inilarawan ng may-akda ang sitwasyon bago ang pananalanta ng bagyo. Ihinalintulad niya ang sitwasyon sa isang hapon na tila isang gabing binalot ng dilim, tahimik na tila ba naghihintay ng pagkakataong sumalakay at tinakpan ang kalangitan at itinago kahit ang liwanag ng mga bituin sa kalangitan. Ang mga ganitong sitwasyon ay isang banta na binalewala ng mga tao.

a.3. Onomatopiya (Onomatopoeia). "Manguli-nguli an piyait han ira kakuri (nakabibingi ang ingay at sigaw ng kanilang paghihirap)”. Ang salitang manguli-nguli sa Waray ay paglalarawan ng isang senaryo ng kaguluhan at maingay na kapaligiran. Ito ay paglalahad ng mga sigaw ng mga biktima habang nananalanta ang bagyo. Isang karima-rimarim na sitwasyon na nais iparinig ng may akda sa kaniyang mga mambabasa. Nariyan ang iba't ibang sigaw ng pagdurusa na tila nakikipagbuno kay kamatayan.

a.4. Pagtawag (Apostrophe). "Idoy, Iday, Hain kamo", Sa tulang "Ikmat sa Dagkon Balud" inilarawan ni Nemesio Baldesco ang sigaw ng katarungan at pagsusumamo ng mga biktima. Marami sa mga biktima ang nawalan ng mga mahal sa buhay at ang pinakamahirap pa ay ilan sa mga ito ay tuluyang nilamon ng higanteng alon ng daluyong at tinangay sa pusod ng karagatan at hindi na nasilayan. Sa tulang ito, ipinakikita ng may-akda ang patuloy na pagsigaw ng mga biktima upang makita, mahanap ang mga labi ng kanilang mga mahal sa buhay.

a.5. Pagpapalit Saklaw (Synecdoche). Isang uri ng matayutay na pagpapahayag na kung saan ay pinapalitan ang katawagan ng isang bagay bilang bahagi ng kabuoan. Binanggit sa tulang "Where U?" ang linyang “pa Bis nimo lambong waray ak kita," isang paglalarawan ng pangungulila sa isang taong mahalaga sa buhay na di na muling nakita pagkatapos ng sakuna. 
a.6. Paralelismo (Parallelism). Ito ay pagpapakita ng serye ng mga ideya/pangyayari na binanggit sa tula. Ginamit ang mga sumusunod na linya: "An ginpaksiw mga tinidor, han gab-i an iginsanlag mga butones, ngan yana nga aga igilugaw an mga timbi" at "Ginlilinabaran, Ginhihinisawan, an monyika nga ira natad-an" upang ipakita na wala sa katinuan ang persona upang gawin ang mga bagay na iyon. Isa lamang ito sa mga naging epekto ng sakuna na may mga biktimang nasiraan ng bait dahil sa sobrang depresiyon dulot ng pangungulila sa mga minamahal sa buhay.

\subsection{Persona}

Kasama sa pagsusuring estruktural ang pagtukoy sa persona ng tula. Layon nitong palutangin ang karakter na nagsasalita sa tula upang mas magkaroon ng malalim na pag-unawa sa mga damdamin at karanasan ng mga biktima. Sa tulang "Noche Buena Ala Yolanda" mahihinuhang biktima ang mismong ang nakaranas ng sakuna (Yolanda Survivor). Makikita sa tula ang mga salitang "Nga amon (na aming)" at "Ginbitay ko (ibibitin ko)", Nagkamata na kami (gumising na kami)." Sa tulang "Gugma, Paglimot Ug Iba pa nga Pinalaypay" ang persona ng tula ay isang tagapagsalaysay sa mga pangyayari na kaniyang naranasan at nasaksihan. Makikita ito sa mga linyang "mahapdusay ha panit (mahapdi sa balat)" na nagpapahiwatig na naranasan din ng may-akda ang lahat ng pangyayari sa kasagsagan ng sakuna. Sa tulang "Yolanda" mahihinuhang ang persona ng tula ay isa ring biktima. Malinaw ang paglalarawan sa tula na tila ba aktuwal na nakikita ang kalagayan at mga pangyayari. Pinatutunayan ito sa unang saknong ng tula. Ang biktima (persona) ang sumisigaw ng pagtawag, "Idoy! Iday! Hain na kamo? Gayundin sa Tulang "Where U?" ang persona ng tula ay isang taong nangungulila sa isang minamahal na hindi na muling nakita. Ipinakikita niya rito ang kaniyang karanasan sa pagbabalewala sa mga taong ito noong mga panahong maaari pa sana niyang ipakita ang kaniyang pagmamahal. Ang persona ay maaaring isang magulang, anak, kasintahan at/o kaibigan. Gayundin, sa tulang "Ha Pagbisita ni Apoy Yolanda" kasamang nakipagsapalaran ang persona ng tula sa hagupit ni Yolanda. Isa siya sa mga biktima at nawalan ng minamahal sa buhay na inilarawan sa tula bilang matalik niyang kaibigan. Marami sa mga tulang naisulat ay gumamit ng persona bilang biktima o survivor. Gayunpaman, may mga tula pa ring gumamit ng mga personang tagapagsalaysay at tagapagmasid lamang. Gaya ng tulang "Ayaw Paurhi, Buligi an Nagkukuri” na kung saan ang persona ay isang tao na naghahangad ng mabilis na pagbangon ng mga biktima sa pamamagitan ng agarang pagtulong at pagdamay.

\subsection{Simbolismo}

Isang magandang lunsaran sa pagsasalaysay ng isang pangyayari ang paggamit ng mga simbolismo. Sa mga tulang naisulat, lumutang sa pagsusuri ng mananaliksik ang sumusunod na simbolismo.

Krismas Tri (Christmas Tree). Ginamit ang mga salitang kahoy, buskay, medyas bilang pagpapakita ng mga materyales na ginamit sa pagbuo ng Krismas Tri. Pagpapahiwatig lamang ito ng pagpapahalaga sa Pasko sa kabila ng hirap na pinagdaraanan.

Piyait (sigaw/hinaing). Ang piyait ay isang representasiyon ng boses ng mga biktima hinggil sa kanilang kalagayan. Inilalarawan sa tula ang hinaing ng mga biktima sa hirap na kanilang dinaranas, pagkawala ng kanilang kabuhayan, pag-aalala at mga pangamba.

Pinalaypay/Linabhan (Mga labahang nakasampay). Ang salitang ito ay ginamit upang kumatawan sa mga lapida ng mga nasawi sa sakuna. Ipinakikita sa tula ang libo-libong lapida ng mga namatay. Pinatutunayan ito sa mga linyang, "Nakit-an han usa nga pasahero: Nakapalaypay an ira mga ngaran, tapad han kanan mga bakwit pinalaypay nga mga linabhan." Ito ay isang matalinhagang pagpapahayag hinggil sa mga naging karanasan ng mga biktima.

Rosaryo, Kaldero, Radyo. Ginamit ang tatlong bagay na ito upang sumimbolo sa tatlong kalagayan na kadalasang nakikita sa oras ng mga sakuna. Kultura na ng mga Pilipino ang pagkapit sa pananampalataya sa pamamagitan ng pananalangin sa mga panahon ng paghihirap. Ang rosaryo na kumatawan sa pananampalataya at pag-asa; ang kaldero na naglarawan sa sitwasyon ng pagkagutom ng mga biktima lalo na sa kasalatan sa pagkain; 
at ang radyo na simbolo ng komunikasyon na sa panahong yaong ay walang maayos na komunikasyon sa lugar na nagdulot ng labis na pagkabahala para sa iilan.

Cellphone. Ang bagay na ito ay kasangkapang pangkomunikasyon, ginamit sa tula ang simbolismong ito upang ipahayag ang kakulangan ng komunikasyon sa mga taong malalapit sa kaniyang puso. Ang bagay na ito ay wika ng dalawang tao na nangangailangan ng pagmamahal.

Bituon (Bituin). Ang bituon ay sagisag ng liwanag at pag-asa, sa gabi ng delubyo ay lilipas din ang makapal na ulap sa kalangitan at muling sisilay ang maningning na mga bituin. Sa panitikang Pilipino, ito ay simbolo ng bagong pag-asa at panimula ng bagong yugto pagkatapos ng isang sakuna.

Iday (Pantawag sa babae). Sa kulturang Waray, ito ay isang katawagan sa anak na babae. Sumisimbolo ito ng kamusmusan, kawalang-malay. Sa tula ipinakikita na si Iday ay biktima ng kapabayaan ng isang inang nawalan ng bait dahil sa sobrang depresiyon at pangungulila sa isang mahal sa buhay.

\subsection{Tema}

Marami sa mga tulang naisulat ay tumalakay sa mga temang pangungulila, paglimot, pagbangon at pagkapit sa mumunting pag-asa. Sa mga temang ito nangibabaw ang tema ng pangungulina na makikita sa mga tulang Yolanda, Where U? at Pag-iliw at Gugma. Sa tulang "Where U?" binanggit ang mga linyang "Intrimis gadla adto nga, Hu U? Baton na gad, Where U?" Ang linyang ito ay pagpapahiwatig ng labis na pangungulila ng isang tao, sa kulturang waray, ang pariralang "na gad" ay isang masidhing damdamin ng pagnanais na makuha o masagot ang isang bagay. Sa linya ring ito ay ipinakikita ang labis na pagsisisi ng isang tao dahil sa pagbalewala o hindi pagseryoso sa mga ugnayan noong nasa ayos pa ang lahat. Sa tulang "Pag-iliw" tinalakay ang kaso ng depresiyon dulot ng labis na pangungulila sa mga mahal sa buhay na nawalay pagkatapos ng sakuna. Dito binibigyan-pansin ang mga epekto nito sa isang tao lalo na sa sikolohikal at pag-iisip. Kapansin-pansin din ang tema ng pangungulila sa tulang "Yolanda." Ang tula ay naglalarawan ng labis na pangungulila sa mga minamahal sa buhay na nawalay dahil sa sakuna. Gumamit ang may-akda ng mga talinghaga na pagtawag (apostrophe) sa linyang, "Idoy, Iday hain kamo" na nagsasaad ng pagdadalamhati at pagluluksa. Gayunpaman, sa kabila ng mga temang ito ay lumutang din ang tema ng kapayapaan at paglimot sa masasamang alaala. Tinalakay sa una at ikalawang saknong ng tulang "Ayaw Paurhi, Buligi an Nagkukuri” ang mapait na karanasan ng mga biktima. Subalit sa mga kasunod na saknong ay ipinahahayag ng tula ay pagnanais na ilibing sa limot ang mga karanasang ito kasama ng mga namayapang kapamilya. Ang positibong pananaw sa buhay ipinapakita sa tulang "Noche Buena Ala Yolanda" ang kahirapan at kasalatan ng buhay pagkatapos ng sakuna. Taliwas man sa nakasanayan, ugaling Pilipino na ano't anupaman, ay nagagawan ng paraan ang lahat ng bagay. Ipinakikita rin sa mga tulang naisulat pagkatapos ng sakuna ang pagkapit sa mumunting pag-asa na inilarawan sa tulang (Ha Pagbisita ni Apoy Yolanda) ang kakayahan ng mga Waray na kumapit sa kahit sa munting pag-asa. Humahanap ng dahilan upang muling buhayin ang lakas ng loob at tibay ng sarili sa kabila ng mga pinagdadaanang hirap sa buhay.

\subsection{Iba't ibang damdamin ng mga tula}

Sa pagsusuri sa damdamin ng mga siday na naisulat pagkatapos ng sakuna, nangingibaw ang damdamin ng kalungkutan. Gayundin, naipakita sa tula ang mga damdamin gaya ng simpatiya, katatagan at pag-asa.

Kalungkutan. Ipinadarama sa tulang "Gugma, Paglimot ngan iba nga Pinalaypay" ang pagdadalamhati at pagluluksa sa pagkawala ng mga mahal sa buhay. Ang linyang "Nakit-an han usa nga pasahero: Nakapalaypay an ira mga ngaran" ay isang pagpapakita ng muling panunumbalik ng sakit at pait ng alaala na mayroon ang sakuna. Gayunpaman, sa kabila ng lahat ng ito ay hindi nawala ang pag-asa sa mga biktima na magkakaroon ng bagong yugto at simula ang kanilang buhay. Pinatutunayan ito sa ikatlong saknong ng tula sa mga mga linyang, "Han nangungunat pa la nga adlaw." Ang liwanag ng adlaw (araw) ay simbolo ng bagong pag-asa para sa mga biktima. Labis na kalungkutan din ang ipinadarama ng tulang "Yolanda" na ipinakikita sa unang linya ng tula at ginamit ang salitang mahaw-ang (mapanglaw). Inilalarawan nito ang kalagayan ng Cancabato (ngayon ay Tacloban) 
pagkatapos ng sakuna. Katahimikan at kalungkutan ang bumalot sa buong lugar pagkatapos ng sakuna. Gayundin, lumutang sa tulang "Where U?" ang pangungulila at panghihinayang at pagsisisi. Sa linyang "Intrimis gadla adto nga, Hu U? Baton na gad, Where U?" ang linyang ito ay pagpapahiwatig ng labis na pangungulila ng isang tao, sa kulturang Waray, ang pariralang "na gad" ay isang masidhing damdamin ng pagnanais na makuha o masagot ang isang bagay. Sa linya ring ito ay ipinakikita ang labis na pagsisisi ng isang tao dahil sa pagbalewala o di pagseryoso sa mga ugnayan noong nasa ayos pa ang lahat.

Simpatiya. Sa mga linya sa ibaba ipinakikita ng may-akda ang pagnanais na matulungan ang mga biktima na gumaan ang bigat ng pasanin na dulot ng sakuna. Hindi lamang materyal na tulong, kundi maging emosyonal at sikolohikal na makikita sa huling linya ng tulang “Ayaw Paurhi, Buligi an Nagkukuri” Ug diri na, mag-antos ngan mahulop pirmi.

Gayundin, sa tulang "Pag-iliw" makikita ang parehong damdamin, awa, pagdamay at pag-asa sa una hanggang ikatlong saknong ipinakikita rito ang pagkaawa ng persona sa kalagayan ng biktima. Kinupkop at inilagaan niya si Iday dahil sa napapabayaan na ito ng kaniyang ina. Isang katangiang natural sa mga Waraynon na mapagmalasakit sa kapuwa lalo na kapag nasa gitna ng mga pagsubok.

Katatagan at Pag-asa. Sa kabila ng mga pagsubok ay laging kumakapit ang mga Waray o mga Pilipino sa kabuoan sa pananampalataya na siyang nagbibigay ng lakas upang bumangon at magpatuloy. Sa huling dalawang linya ng tulang "Ha Pagbisita ni Apoy Yolanda" makikita rito ang pagkapit sa panalangin at pagsibol ng pag-asa. Binanggit ang, "Buhat na gad! Buhat na gad!" Sa tulang "Noche Buena Ala Yolanda" ipinadarama ng tula ang pagtitiis at pag-asa na nagpapakita ng pigiging matiisin at masinop ng mga Waray. Sa kabila ng trahedya, kalungkutan at pangungulila ay nagagawa pa rin nilang ipagdiwang ang isang mahalagang okasyon sa relihiyong Katoliko na sumisimbolo ng katatagan, pag-asa at pananampalataya.

\subsection{Iba't ibang karanasan pagkatapos ng sakuna}

Kahikahusan, Pagkakaisa, at Pagiging Matiyaga. Maraming karanasan ang ginamit sa mga tula upang ipakita ang karanasan ng pagbangon ng mga biktima pagkatapos ng sakuna. Isa na rito ang kahikahusan na ipinakita sa larawan ng Krismas Tri na gawa sa mga kahoy, medyas at buskay na pinulot lamang. Ang gayong paaglalarawan ay malinaw na manipestasyon ng kahikahusan ng mga biktima. Kapansin-pansin din ang ugali ng pagkakaisa sa gitna ng mga sakuna tulad na lamang ng mga binanggit sa tula: Ginpatindog ni Manoy an sanga sito kahoy nga amon ginbayawan. Ang salitang amon at ginbayawan ay mga salitang nagsasaad ng pagdadamayan upang bumangon sa kabila ng mga trahedya. Ang pagiging matiyaga rin ay tahasang ipinapakita sa tula sa mga linyang: An gintirok nga buskay na salamin ng pagiging kontento sa mga bagay na nariyan, Ginbitay ko an medyas nga naluho pagbaktas, linya mula sa tulang "Noche Buena Ala Yolanda" na naglalarawan ng karanasan ng mga biktima na maging kontento sa kung ano ang mayroon sila at wala.

Labis na pagdadalamhati, kalungkutan at pagkabalisa. Sa tulang Where u? ay binanggit ang mga linyang Ginhiling ko ngatanan nga iskinita, bis nimo lambong waray pa ak kita, linmabay na an pakwarenta, inim ngaran waray man ha lista, ang mga linyang ito paglalarawan ng karanasan ng mga biktima ng pagdadalamhati sa mga namatay na mahal sa buhay dulot ng Yolanda. Gayundin, sa tulang Yolanda, binanggit ang mga linyang, Gin-iinop ko la ikaw; ha panangis han mga bata ha pinamatinan, ang pagdadalamhati ng mga biktima ay lantad sa linyang ito na kung saan ay sa panaginip na lamang nila nakikita ang mga kanilang mga mahal sa buhay. Inilalarawan din sa tulang "Pag-iliw" ang karanasan ng paghagupit ni Yolanda, ang paglakas ng hangin na halos liparin ang anumang nakadikit sa lupa, paglakas ng ulan, pagbaha, pagdantay ng daluyong sa lupa tangay ang mga higanteng barko at nag-iwan ng libo-libong bangkay. Ang lahat ng ito ay isang mapait at masamang alaala, karanasang di malilimutan at nakaukit na sa kasaysayan. Ngunit sa kabila ng lahat ng ito, nanatiling matatag ang pananampalataya at paniniwala nila na nagbigay sa kanila ng bagong pag-asa. Gayundin, makikita sa tulang "Gugma, Paglimot ngan iba nga Pinalaypay" ang linyang "mahapdusay ha panit" na isang talinghaga na naglalarawan ng kapaitan ng buhay sa kasagsagan ng sakuna mahapdusay ha panit (masakit sa balat) ay isang 
paglalarawan ng malalim na damdamin ng pagtitiis.

Pagtutulungan. Sa unang dalawang saknong ng tulang "Ayaw Paurhi, Buligi an Nagkukuri” ay ipinakikita ang hirap na pinagdaraanan ng mga biktima. Sumisigaw sila sa hinagpis at pagluluksa dahil sa pagkasira ng mga buhay at ng kanilang kabuhayan. Gayunpaman, inilalarawan sa tula ang pagsusumamo na unahing tugunan ang mga pangangailangan ng mga biktima upang sila'y makabangon at mawala ang anumang pagkabahala sa kinabukasan at kapalaran. Patunay ditto ang linyang Ah! Pabay-i na, dali-a lugod assay hira buligi. Sa tulang "Noche Buena Ala Yolanda", ginamit ang mga salitang ginbayawan at nagkamata na kami na nagpapahiwatig ng mga kilos na gawa dalawa o higit pang bilang ng tao upang maisakatuparan ang isang gawain.

Aktuwal na pagdanas sa sakuna. Sa mga tulang naisulat pagkatapos ng sakuna ay naipakita ang karanasan ng mga biktima sa aktuwal na pakikipagbuno sa hagupit ng bagyo. Sa unang linya ng tulang "Yolanda" "Mahang-aw sa Cancabato" ay ipinakita ng may-akda na binalot ng katahimikan ang buong lugar. Inilalarawan din sa tula ang mga bangkay ng mga biktima na nilamon ng tubig, makikita ito sa ikatlong linya na "Maaringasaw sa Suhi." Gayundin, ang pagdanas ng persona sa nakabibinging sigaw ng mga biktima sa sobrang pagdadalamhati at pagdurusa ay isang malinaw na larawan sa tula na ipinikikita sa linyang, "Naaringawngaw an buhi."

Karanasan ng Pagsisisi. Malinaw sa mga linya ng tulang "Where U?" ang panghihinayang at pagsisi ng persona sa pagsasawalang-bahala sa kahalagahan ng pagpapakita ng pagmamahal.

\section{Konklusyon at rekomendasyon}

Napatunayan sa pag-aaral na ito ang sumusunod:

\section{Sa estruktura:}

> Ang mga tayutay na ginamit sa tula ay Pagbibigay Katauhan, Pagtutulad, Onomatopiya, Pagmamalabis, Pagtawag, Pagpapalit-Saklaw at Paralelismo. Higit nitong binigyang anyo ang tula na mas nagpatingkad sa kulay at rikit ng bawat piyesa.

> Marami sa siday naisulat pagkatapos ng sakuna ay gumamit ng persona ng pagiging biktimang aktuwal na dumanas ng kahirapan sa gitna ng delubyo, mayroon ding persona ng survivor, mga tagapagsalaysay ng mga pangyayari lamang at tagamasid na nagmamalasakit sa kapakanan ng mga biktma.

> Lumutang din sa mga siday na ito ang mga simbolong Krismas Tri, Piyait (sigaw/hinaing), Pinalaypay/Linabhan, Rosaryo, Kaldero, Radio, Cellphone, Bituon, at Iday. Lahat ay may kaniya-kaniyang kahulugan at kumakatawan sa iba’t ibang pangyayari.

> Naging pangunahing tema ng mga siday ang pagluluksa, pagdadalamhati, pagdurusa at pagkakaroon ng pag-asa. Ang mga damdaming nakapaloob sa tula ay kalungkutan, simpatiya, katatagan at pag-asa. Sa huli, nalaman ng mananaliksik ang karanasan ng kahikahusan, pagkakaisa, at pagiging matiyaga, labis na pagdadalamhati, kalungkutan at pagkabalisa, pagtutulungan, aktuwal na pagdanas sa sakuna at pagsisisi.

Batay sa mga datos na nakuha, nahinuha ng mananaliksik na ang mga siday ay naglalaman ng masidhing damdamin at karanasan ng paghihirap at pagbangon. Ang mga damdaming ito ay kalungkutan, katatagan, at pag-asa.

Ang mga karanasang nakapaloob sa mga siday ay kahikahusan, pagkakaisa, at pagiging matiyaga, pagdanas ng labis na pagdadalamhati, kalungkutan at pagkabalisa, pagtutulungan, aktuwal na pagdanas sa sakuna, at karanasan ng pagsisisi. 


\section{Inirerekomenda sa pag-aaral na ito ang sumusunod:}

$>\quad$ Magsagawa ng pag-aaral sa pagsusuri ng mga tula na nakatuon sa kontekstong kultural ng mga Waray hinggil sa mga paraan ng pagbangon sa mga sakuna.

$>\quad$ Magkaroon ng mas masusing pag-aaral sa pag-uugnay ng buhay ng may-akda sa kaniyang mga akda upang mas makita ang awtentisiti ng mga karanasan at damdamin ng mga tulang naisulat pagkatapos ng sakuna.

\section{Sanggunian}

Alegre, E. N., \& Fernandez, D. G. (1987). Writers and their milieu: An oral history of second generation writers in English [Di-nalathalang disertasyon]. De la Salle University, Manila, Philippines.

Arsolon, V. A. (2012). El Filibusterismo ni Rizal: Mimesis na sosyo-politikal [Di-nalathalang tesis]. Cebu Normal University, Cebu City.

Catipay, T. A. (2013). Corazon, ang unang aswang ni Richard Somes: Katauhan, lipunan, balik-sulat [Di-nalatahalang tesis]. Cebu Normal University, Cebu City.

Cericos, E. U. (2011). Pagsusuri sa mga bisang pampanitikan ng tula [Di-nalatahalang tesis]. Cebu Normal University, Cebu City.

Egoy, C. J., Arce, S. A., Colasito, J. C., Quimbo, L. P., \& Villamor, M. M. (2019). Ugnayang kultura at panitikan: Pagsusuri sa mga pagpapahalagang kultural na nakapaloob sa akdang Inintokan ni Victor N. Sugbo [Di-nalathalang tesis]. Leyte Normal University, Tacloban City.

Gleeson, P. (2018). Mimetic theory of literary criticism. Retrieved from https://penandthepad.com/mimetic-theory-literary-criticism-5761846.html

Leyte-Samar Heritage Society, Inc. (2015). Lunop: Haiyan voices and images. Tacloban City: Leyte Samar Heritage Society.

Macan, R. S. (2000). Mga konseptong maka-Diyos, makatao at makakalikasan sa mga piling romantikong tula ni Jose Corazon de Jesus [Di-nalatahalang disertasyon]. Cebu Normal University, Cebu City.

Mambrol, N. (2016). Literary theory and criticism. Retrieved from https://www.google.com/amp/s/literariness.org/2016/03/20/structuralism/amp/

Ranada, P. (2013). Yolanda/Haiyan damage now at 9.46B. Retrieved from https://www.rappler.com/move-ph/issues/disasters/typhoon-yolanda/43858-20131116-yolanda-death-tol 1-ndrrmc-am-report

Redmond, M. V. (2015). Symbolic interactionism: English technical report and white papers. Retrieved from http://lib.dr.iastate.edu/engl_reports/4

Rio, M. T. (2013). Kamalayang pangmamamayan sa mga piling tula ni Amado V. Hernandez [Di-nalatahalang tesis]. Cebu Normal University, Cebu City.

San Juan, G. P. (1995). Pantikang Pilipino. Quezon City: Katha Publishing Company.

Santiago, E. (1989). Panitikang Pilipino: Kasaysayan at pag-unlad pangkolehiyo. Manila, Philippines: National Bookstore.

Villafuerte, P. V. (2000). Panunuring pampanitikan. Valenzuela City: Mutya Publishing House. 